\title{
Ischemic bowel secondary to ventriculoperitoneal shunt knotting: a surprisingly rare complication of ventriculoperitoneal shunting. Case report
}

\author{
${ }^{*}$ Kathryn Alana Joy, MD, ${ }^{2}$ Benjamin Stephan Szewczyk, MD, ${ }^{1}$ Matthew Armand Adamo, MD, ${ }^{1}$ and \\ Mary Christina Whyte, MD²
}

Departments of ${ }^{1}$ Neurosurgery and ${ }^{2}$ Pediatric Surgery, Albany Medical Center, Albany, New York

Small-bowel obstruction (SBO) and volvulus as a result of ventriculoperitoneal shunting are a rare phenomenon, especially when resulting in bowel necrosis. The authors report the rare event of SBO, bowel strangulation, and necrosis in a pediatric patient after the abdominal catheter became knotted around his small bowel, and they provide a comprehensive review of the literature. The authors argue that shunt configuration is an important consideration for a patient presenting with $\mathrm{SBO}$, and that symptomatic shunt knotting is a reason for surgical correction.

https://thejns.org/doi/abs/10.3171/2019.11.PEDS19138

KEYWORDS small-bowel obstruction; SBO; ischemic bowel; VPS; knot; ventriculoperitoneal shunt complication; hydrocephalus

$\mathrm{V}$ ENTRICULOPERITONEAL shunts (VPSs) are the most common form of CSF diversion system in use, and thousands are placed in the United States each year. Common complications include infection, malfunction, and over- and underdrainage, as well as abdominal complications including peritonitis, adhesions, herniation, migration, bowel perforation, pseudocyst formation, and small-bowel obstruction (SBO) or volvulus. SBO and volvulus as a complication of VPS remain an extremely rare phenomenon, with only 23 cases reported in the literature; moreover, SBO and volvulus associated with bowel gangrene and necrosis as a complication of VPS remain exceedingly rare, with only 8 cases reported. We report the case of a 5-year-old boy presenting in extremis who was found to have SBO with necrotic bowel secondary to an abdominal VPS knot, and we have also performed a systematic review of the literature. We demonstrate the presence of a knot on shunt radiographs obtained over several years, which should be noted even in an asymptomatic patient. VPS configuration should be well noted on shunt radiographs, and the presence of a suspicious knot or coil should lower the threshold for operative intervention if the patient is clinically symptomatic.

\section{Methods}

The case was reviewed by the IRB committee and a release form for imaging and photography was signed by the underage patient's healthcare proxy. Imaging was obtained and annotated from the internal PACS. A photograph of the intraoperative findings was obtained. The literature review was completed using PubMed.gov and Google Scholar including the following search terms: "abdominal complications of ventriculoperitoneal shunt," "small bowel obstruction from ventriculoperitoneal shunt," and "volvulus from ventriculoperitoneal shunt." Any article related to SBO or volvulus as a result of VPS was included as well as reported knot/coil formation of any kind.

\section{Case Report}

History

We present the case of a 5-year-old boy who presented with a 1-day history of worsening lethargy and emesis. He had a past medical history significant for grade 4 intraventricular hemorrhage in infancy with a VPS status post multiple revisions (the last of which was placed via minilaparotomy at another institution), as well as a history 


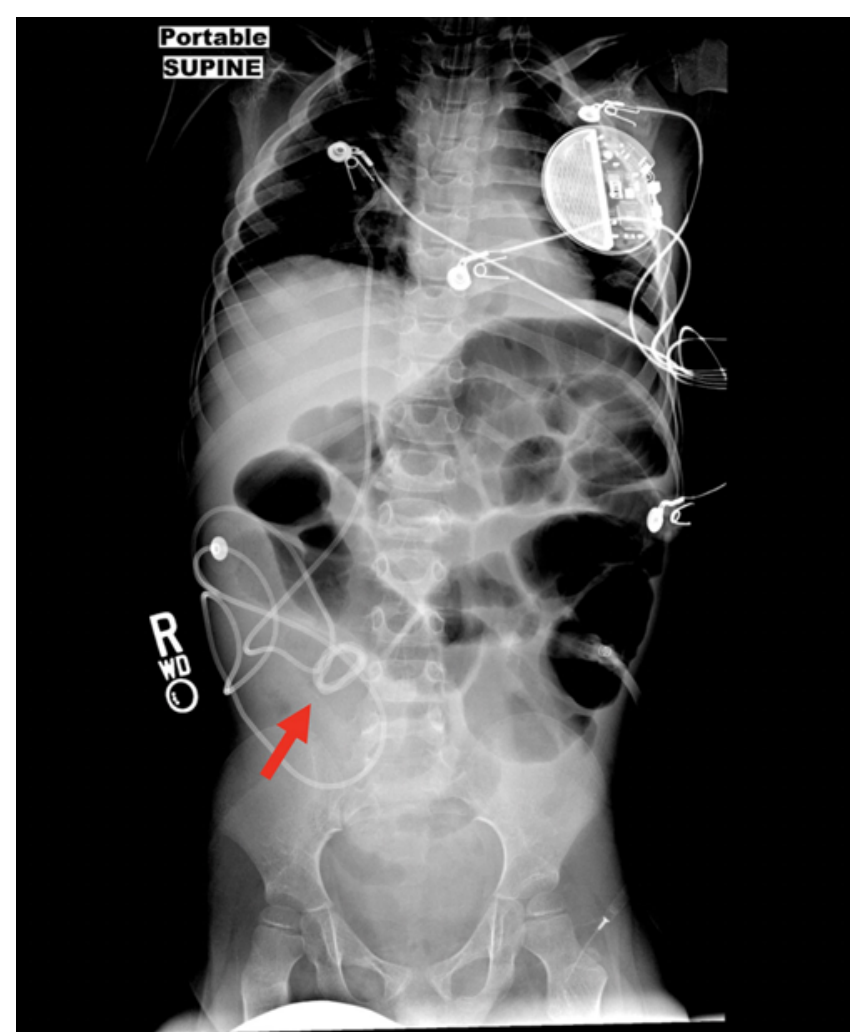

FIG. 1. Abdominal radiograph obtained on presentation showing dilated loops of small bowel and concern for SBO. The VPS knot was visible in the patient's right midabdomen (red arrow).

of Nissen fundoplication, pyloroplasty, G-tube placement, and vagal nerve stimulator placement for medically refractory epilepsy. The patient had presented 1 year previously with a bowel obstruction that had been successfully treated with conservative management.

\section{Examination}

On arrival to the emergency department the patient was found to have agonal respirations and received emergency intubation. He underwent an emergency CT head and shunt series to evaluate for shunt failure, but the shunt was found to be functioning appropriately with no increase in ventricular size. On examination the patient was noted to have a markedly distended but compressible abdomen, and the shunt series abdominal film was significant for severely dilated bowel and concern for SBO (Fig. 1). On laboratory examination the patient was found to have an acute kidney injury and a profound leukocytosis of 53,700/ $\mu \mathrm{l}$ with a procalcitonin of $8.23 \mathrm{ng} / \mathrm{ml}$. A CT abdomen-pelvis sequence was performed, which showed a large amount of free fluid with severely dilated bowel with focal areas of decompression and no obvious transition point.

\section{Operation}

The patient was taken on an emergency basis to the operating room for an exploratory laparotomy. On inspection of the bowel it was noted that several loops of small bowel had been tied into a bouquet configuration by the VPS

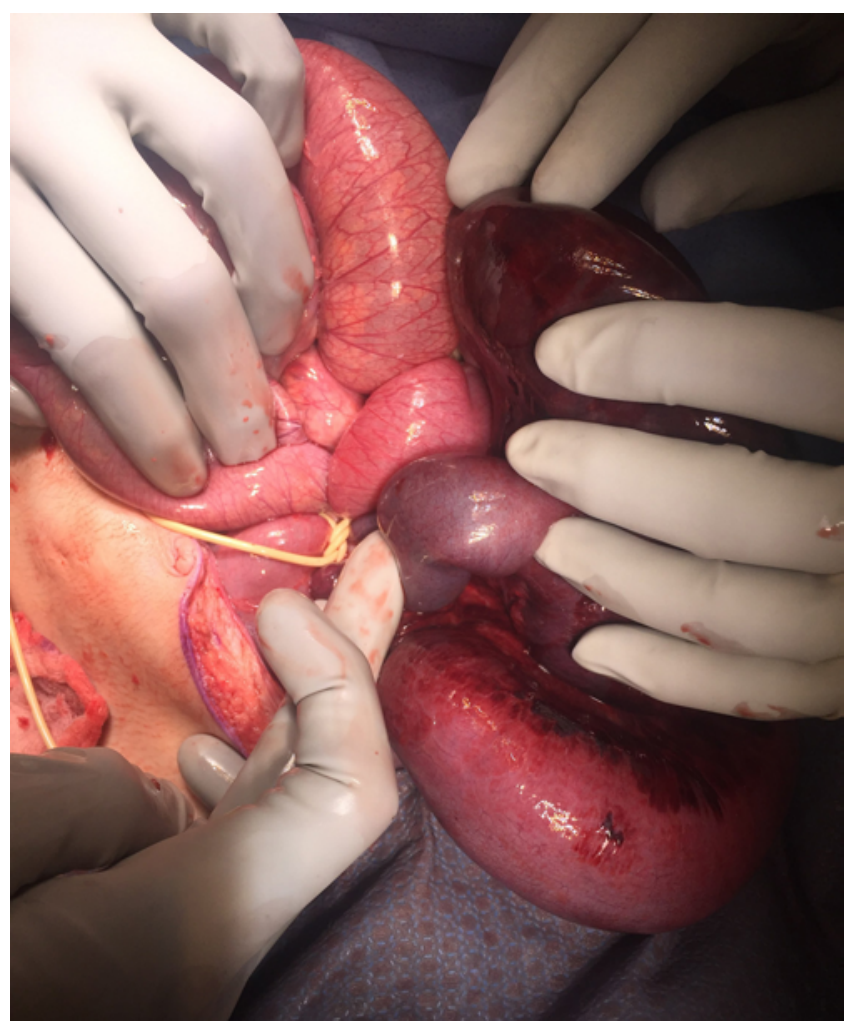

FIG. 2. Intraoperative photograph of small bowel strangulated by the VPS catheter knot.

catheter, which was found to be tightly knotted (Fig. 2). Approximately $60 \mathrm{~cm}$ of small bowel was noted to be necrotic and was ultimately resected. The patient's VPS was externalized, and he was left in discontinuity with plans to return to the operating room for closure the following day. At his second operation, another $15 \mathrm{~cm}$ of terminal ileum was found to be necrotic and nonviable and he underwent an ileocecectomy with an ileocolonic anastomosis.

\section{Postoperative Course}

The patient was continued on broad-spectrum antibiotics in the interim and his shunt was reinternalized in the abdomen (since there was no active infection or history of nonabsorption) on postoperative day 12 . He had an otherwise uncomplicated hospital course and was discharged home on hospital day 16.

\section{Discussion}

Thousands of VPSs are placed every year in the United States, ${ }^{20}$ but bowel obstruction secondary to strangulation by the shunt catheter remains a rare complication. Shunt complications can include infection, proximal malfunction, disconnected tubing, distal clogging, shortening, and over- and underdrainage. Abdominal complications can include postoperative peritonitis, adhesions, herniation, migration, bowel perforation, pseudocyst formation, and bowel obstruction or volvulus. ${ }^{5}$ SBO from shunted hydrocephalus remains a rare phenomenon, with 23 cases 
reported in the literature. . $^{-3,5,6,9-12,14,17,18,21-27,29,31}$ SBO or volvulus secondary to VPS that involves bowel strangulation or necrosis is an even more rare phenomenon, with only 8 cases reported in the literature. ${ }^{12,15,22,25,26,29,31}$ Knot or coil formation in the distal portion of the VPS has been reported $^{4-10,12-19,22-28,30}$ mainly as an iatrogenic event after an attempt at externalizing the abdominal shunt tubing. ${ }^{12,22,}$ ${ }^{25,26}$ In 1 case, after meeting resistance while removing the distal catheter and finding it to be knotted in the abdomen, Sanan et al. were able to relieve the knot by using intraoperative fluoroscopy and an angiographic guidewire. ${ }^{22}$ Proposed mechanisms for spontaneous knot formation include crowding of the intraabdominal contents (such as in pregnancy), small diameter and high elasticity of the tubing, intense bowel peristalsis, or excessive intraperitoneal catheter length, ${ }^{6,7,15,16}$ although some authors believe that spontaneous intraabdominal knot formation is not possible. ${ }^{10}$ Table 1 includes a comprehensive list of all reported bowel obstruction/volvulus and knot/coil formation cases and their relationship to one another as well as whether this was a reportedly iatrogenic event.

In our case we believe there are several possible explanations for how this event may have happened. Proposed mechanisms (described above) for knot formation include iatrogenic events such as pulling the distal portion and leaving this in place when resistance is met. We believe that this is unlikely to be the case because the shunt in this patient is in complete continuity based on the imaging, with no retained catheter fragments indicative of this complication. Other proposed risk factors include crowding of the intraabdominal contents (such as in pregnancy), small diameter and high elasticity of the tubing, intense bowel peristalsis, or excessive intraperitoneal catheter length. The patient did have several prior abdominal surgeries including multiple shunt revisions, Nissen fundoplication, pyloroplasty, and G-tube placement. Prior surgeries can lead to abdominal adhesions, which effectively compartmentalizes the abdomen and leads to worse crowding of the intraabdominal contents. The last revision was when the patient was 2 years old, and because it is common practice to place adequate catheter length for the patient to grow, excessive length may have been left during that time. It is possible that a combination of excessive catheter length, crowding of the abdominal contents, and peristalsis over several years could have led to the catheter becoming knotted. It could have also been slightly coiled when placed originally, and that could have been exacerbated over several years of growth and peristalsis.

The best way of preventing the shunt knot/coil formation would be to take anticipatory steps toward avoiding the aforementioned risk factors such as direct visualization of placement of the abdominal catheter or by routinely obtaining a postoperative shunt series. For example, in a patient with multiple prior abdominal surgeries, it would be reasonable to perform shunt revision in conjunction with the general surgery team and to place the distal catheter via direct laparoscopic observation. Given our patient's history, it may have been reasonable for the last revision to have been done in this way rather than via minilaparotomy. Other ways of preventing a knot in the acute setting of shunt revision would to be to take active steps in troubleshooting the operation if resistance is met. For example, if resistance is met when trying to remove the catheter it would be reasonable to call an immediate intraoperative general surgery consultation for assistance or to take a radiograph and observe the configuration. If the radiograph looks concerning for knotting, then it may be reasonable to convert the abdominal operation to laparoscopic or open surgery, or to try unknotting the catheter under fluoroscopic guidance as described by Sanan et al. This is done by taking a stiff angiographic guidewire and slowly introducing it into the lumen in an attempt to uncoil the catheter and allow it to be removed by gentle traction. ${ }^{22}$

On review of the imaging from the patient's previous hospital visits it was apparent that the knot in his peritoneal catheter had been there for some time, even 3 years prior on a shunt series obtained after breakthrough seizures (Figs. 3 and 4). To the best of our knowledge, our case report is the only one documented with serial imaging concerning for impending bowel strangulation. This makes the argument that continuity and location of the shunt catheter is not the only important consideration in a shunt series, but also the configuration. In general, we believe that imaging should be obtained if a patient demonstrates signs of shunt failure or bowel obstruction or perioperatively. We do not advocate for routine serial imaging; we would obtain serial imaging only if the patient is symptomatic and following shunt revision, to provide a new baseline and ensure that the shunt is contiguous, in the correct cavity, and has a normal configuration. Bowel obstruction with a concerning shunt configuration should lower the threshold for operative intervention, even in the absence of an acute abdomen. It would have been reasonable to electively correct the knotted distal catheter during the first episode of SBO 1 year previously if it had been recognized at that time. We believe a knot or coil with evidence of SBO or volvulus warrants shunt exploration and revision because it may prevent a potentially life-threatening event in the future. An algorithm to manage a discovered knot/coil would be to assess the patient's symptoms: If asymptomatic, observation should be used. If there are signs of shunt failure, the cranial component should be explored with testing of proximal and distal flow. If there is proximal failure, the proximal catheter should be replaced. If there is distal failure, or if the patient presents with symptoms of bowel obstruction, then exploration of the abdominal component should be performed with replacement or revision of the distal catheter.

\section{Conclusions}

Fortunately, bowel obstruction and subsequent ische$\mathrm{mia} /$ necrosis secondary to VPS catheter strangulation remain an extremely rare phenomenon. We advocate that bowel obstruction with an abdominal VPS catheter should prompt assessment of the configuration of the catheter, particularly looking for the presence of any knotting or coiling on imaging. The presence of a knot or coil in the setting of bowel obstruction is an indication for surgical correction because it could potentially prevent a future intraabdominal catastrophe. 


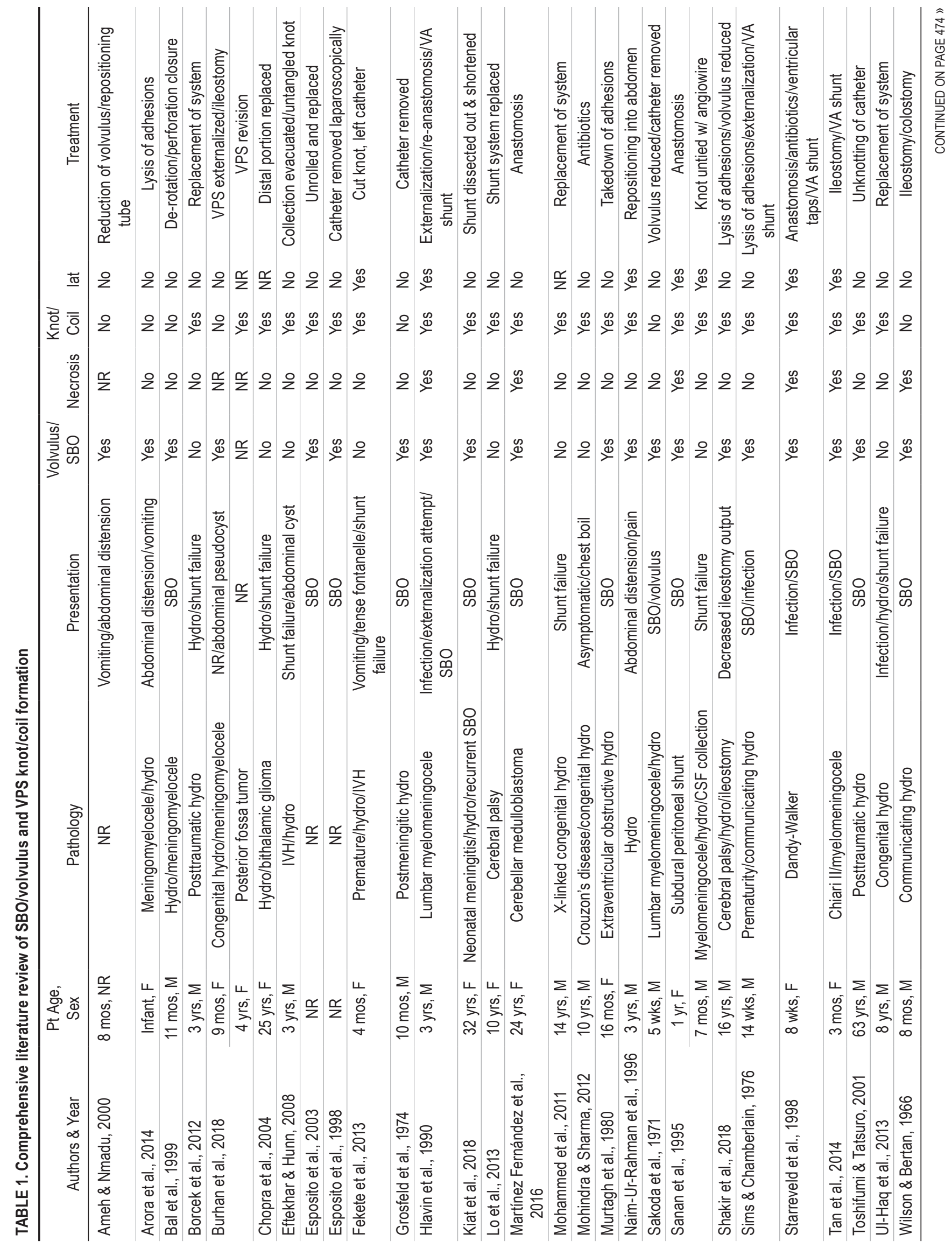




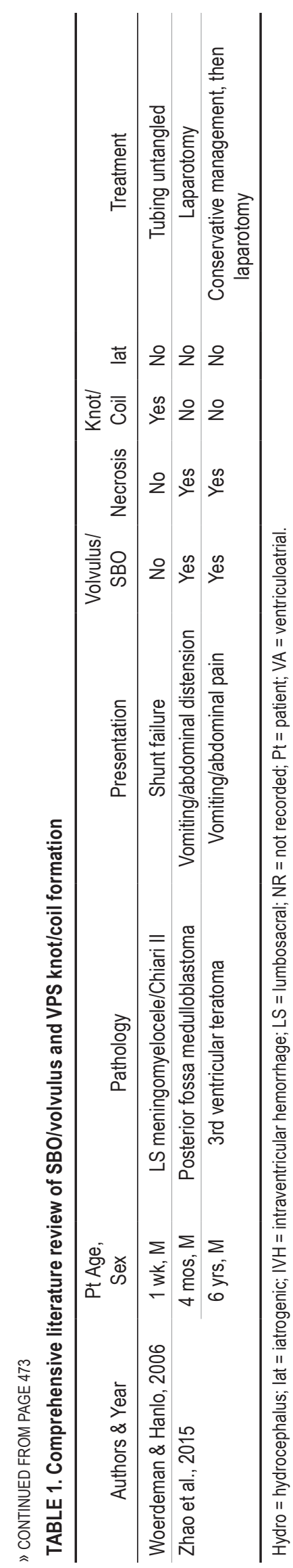

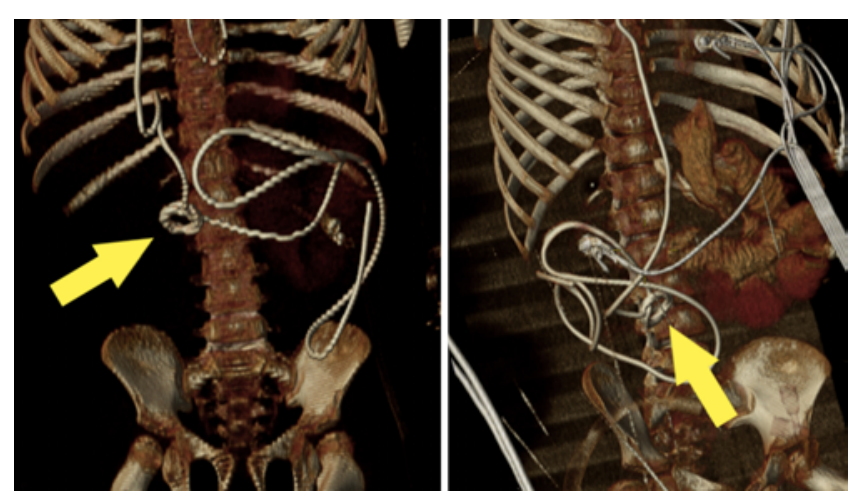

FIG. 3. 3D reconstructions of CT scans from the patient's sentinel bowel obstruction 1 year prior to current presentation (2017; left) and from the patient's most recent admission (2018; right). The knot in the catheter is highlighted with arrows.
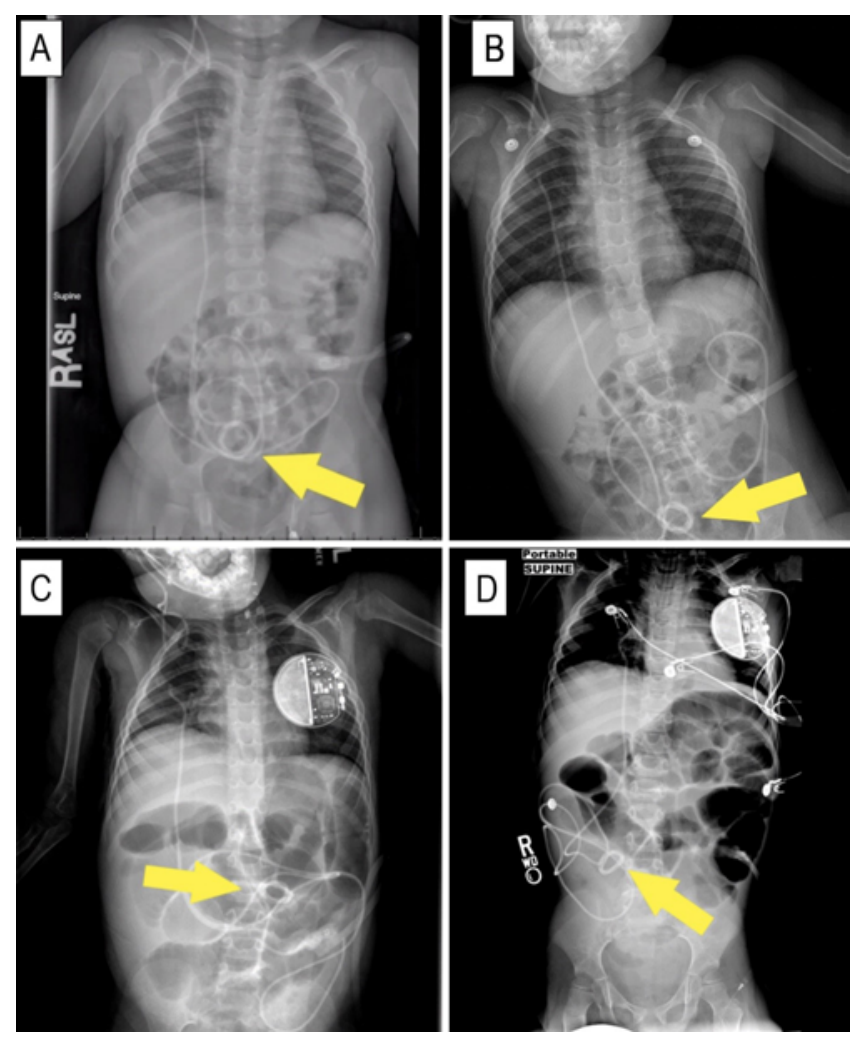

FIG. 4. Shunt series and abdominal radiographs spanning 3 years demonstrating a persistent knot in the VPS catheter. Knot is highlighted with arrows. A: Shunt series (8/5/15). B: Shunt series (3/21/17). C: Abdominal radiograph evaluating for obstruction (8/24/17). D: Abdominal radiograph obtained during patient's current admission (8/24/17).

\section{References}

1. Ameh EA, Nmadu PT: Intestinal volvulus: aetiology, morbidity, and mortality in Nigerian children. Pediatr Surg Int 16:50-52, 2000

2. Arora P, Autkar GM, Kumar A, Pawar SS, Gutte AA: Intestinal obstruction due to adhesions around VP shunt tip in a case of pyogenic meningitis. OMICS J Radiol 3:172, 2014

3. Bal RK, Singh P, Harjai MM: Intestinal volvulus - a rare 
complication of ventriculoperitoneal shunt. Pediatr Surg Int 15:577-578, 1999

4. Borcek AO, Civi S, Golen M, Emmez H, Baykaner MK: An unusual ventriculoperitoneal shunt complication: spontaneous knot formation. Turk Neurosurg 22:261-264, 2012

5. Burhan B, Serdar KB, Abdurrahman A, Edip AM, Ebuzer D: Abdominal complications of ventriculoperitoneal shunt in pediatric patients: experiences of a pediatric surgery clinic. World Neurosurg 118:e129-e136, 2018

6. Chopra I, Gnanalingham K, Pal D, Peterson D: A knot in the catheter - an unusual cause of ventriculo-peritoneal shunt blockage. Acta Neurochir (Wien) 146:1055-1057, 2004

7. Eftekhar B, Hunn A: Ventriculoperitoneal shunt blockage due to spontaneous knot formation in the peritoneal catheter. Case report. J Neurosurg Pediatr 1:142-143, 2008

8. Esposito C, Colella G, Settimi A, Centonze A, Signorelli F, Ascione G, et al: One-trocar laparoscopy: a valid procedure to treat abdominal complications in children with peritoneal shunt for hydrocephalus. Surg Endosc 17:828-830, 2003

9. Esposito C, Porreca A, Gangemi M, Garipoli V, De Pasquale M: The use of laparoscopy in the diagnosis and treatment of abdominal complications of ventriculo-peritoneal shunts in children. Pediatr Surg Int 13:352-354, 1998

10. Fekete G, Nagy A, Pataki I, Bognar L, Novák L: Shunt insufficiency due to knot formation in the peritoneal catheter. Ideggyogy Sz 66:277-279, 2013

11. Grosfeld JL, Cooney DR, Smith J, Campbell RL: Intra-abdominal complications following ventriculoperitoneal shunt procedures. Pediatrics 54:791-796, 1974

12. Hlavin ML, Mapstone TB, Gauderer MW: Small bowel obstruction secondary to incomplete removal of a ventriculoperitoneal shunt: case report. Neurosurgery 26:526-528, 1990

13. Kiat A, Wright DB, Rebello IG: Intraperitoneal knotting of a ventriculoperitoneal shunt causing small bowel obstruction in an adult. ANZ J Surg 88:E71-E72, 2018

14. Lo WB, Ramirez R, Rodrigues D, Solanki GA: Ventriculoperitoneal shunt disconnection associated with spontaneous knot formation in the peritoneal catheter. BMJ Case Rep 2013:1-3, 2013

15. Martínez Fernández J, Guillén Paredes MP, Lirón Ruiz RJ: [Ventriculo-peritoneal catheter knot with obstruction and intestinal necrosis: an unusual and serious complication.] Med Clin (Barc) 147:471-472, 2016 (Spanish)

16. Mohammed W, Wiig U, Caird J: Spontaneous knot; a rare cause of ventriculoperitoneal shunt blockage. Br J Neurosurg 25:113-114, 2011

17. Mohindra S, Sharma M: Spontaneous knotting of peritoneal catheter: a report of an asymptomatic patient. J Pediatr Neurosci 7:151-153, 2012

18. Murtagh FR, Quencer RM, Poole CA: Extracranial complications of cerebrospinal fluid shunt function in childhood hydrocephalus. AJR Am J Roentgenol 135:763-766, 1980

19. Naim-Ur-Rahman, Jamjoom A, Jamjoom ZAB: Intestinal obstruction caused by extraperitoneal cerebrospinal fluid collection. Pediatr Neurosurg 25:160-163, 1996

20. Patwardhan RV, Nanda A: Implanted ventricular shunts in the United States: the billion-dollar-a-year cost of hydrocephalus treatment. Neurosurgery 56:139-145, 2005

21. Sakoda TH, Maxwell JA, Brackett CE Jr: Intestinal volvulus secondary to a ventriculoperitoneal shunt. Case report. J Neurosurg 35:95-96, 1971
22. Sanan A, Haines SJ, Nyberg SL, Leonard AS: Knotted bowel: small-bowel obstruction from coiled peritoneal shunt catheters. Report of two cases. J Neurosurg 82:1062-1064, 1995

23. Shakir AK, Ramji F, Shakir B, Prabhu S, Kumar R, Altaf MA: If it's not broken, should we fix it? Ventriculoperitoneal shunt catheter contributing to closed-loop small bowel obstruction in a pediatric patient with ileostomy. Pediatr Neurosurg 53:209-210, 2018

24. Sims DG, Chamberlain J: Small bowel obstruction following the insertion of a ventriculoperitoneal shunt. J R Coll Surg Edinb 21:109-111, 1976

25. Starreveld Y, Poenaru D, Ellis P: Ventriculoperitoneal shunt knot: a rare cause of bowel obstruction and ischemia. Can J Surg 41:239-240, 1998

26. Tan LA, Kasliwal MK, Moftakhar R, Munoz LF: Ventriculoperitoneal shunt with a rare twist: small-bowel ischemia and necrosis secondary to knotting of peritoneal catheter. J Neurosurg Pediatr 14:234-237, 2014

27. Toshifumi SNK, Tatsuro I: [Intestinal obstruction caused by ventriculoperitoneal shunt knot. A case report.] J Iwate Med Assoc 53:43-46, 2001 (Japanese)

28. Ul-Haq A, Al-Otaibi F, Alshanafey S, Sabbagh MD, Al Shail E: Ventriculoperitoneal shunt peritoneal catheter knot formation. Case Rep Neurol Med 2013:628493, 2013

29. Wilson CB, Bertan V: Perforation of the bowel complicating peritoneal shunt for hydrocephalus. Report of two cases. Am Surg 32:601-603, 1966

30. Woerdeman PA, Hanlo PW: Ventriculoperitoneal shunt occlusion due to spontaneous intraabdominal knot formation in the catheter. Case report. J Neurosurg 105 (3 Suppl):231-232, 2006

31. Zhao R, Shi W, Yu J, Gao X, Li H: Complete intestinal obstruction and necrosis as a complication of a ventriculoperitoneal shunt in children: a report of 2 cases and systematic literature review. Medicine (Baltimore) 94:e1375, 2015

\section{Disclosures}

The authors report no conflict of interest concerning the materials or methods used in this study or the findings specified in this paper.

\section{Author Contributions}

Conception and design: all authors. Acquisition of data: all authors. Analysis and interpretation of data: all authors. Drafting the article: all authors. Critically revising the article: all authors. Reviewed submitted version of manuscript: all authors. Approved the final version of the manuscript on behalf of all authors: Szewczyk. Administrative/technical/material support: Adamo, Whyte. Study supervision: Adamo, Whyte.

\section{Correspondence}

Benjamin Stephan Szewczyk: Albany Medical Center, Albany, NY.szewczb@amc.edu. 